\title{
Analysis of energy resolution in the KURRI-LINAC pulsed neutron facility
}

\author{
Tadafumi Sano $^{1 \text {, a }}$, Jun-ichi Hori ${ }^{1}$, Yoshiyuki Takahashi ${ }^{1}$, Hiroshi Yashima ${ }^{1}$, Jaehong Lee $^{2}$, and Hideo Harada ${ }^{3}$ \\ 1 Kyoto University Research Reactor Institute, Asashiro-Nishi, Kumatori-cho, Sennan-gun, Osaka 590-0494, Japan \\ 2 Department of Nuclear Engineering, Kyoto University, Kyoto University Katsura, Nisikyo-ku, Kyoto 615-8540, Japan \\ 3 Japan Atomic Energy Agency, Tokai-mura, Naka-gun, Ibaraki 319-1195, Japan
}

\begin{abstract}
In this study, we carried out Monte Carlo simulations to obtain the energy resolution of the neutron flux for TOF measurements in the KURRI-LINAC pulsed neutron facility. The simulation was performed on the moderated neutron flux from the pac-man type moderator at the energy range from $0.1 \mathrm{eV}$ to $10 \mathrm{keV}$. As the result, we obtained the energy resolutions $(\Delta \mathrm{E} / \mathrm{E})$ of about $0.7 \%$ to $1.3 \%$ between $0.1 \mathrm{eV}$ to $10 \mathrm{keV}$. The energy resolution obtained from Monte Carlo simulation agreed with the resolution using the simplified evaluation formula. In addition, we compared the energy resolution among KURRI-LINAC and other TOF facilities, the energy dependency of the energy resolution with the pac-man type moderator in KURRI-LINAC was similar to the J-PARC ANNRI for the single-bunch mode.
\end{abstract}

\section{Introduction}

The electron linear accelerator at the Research Reactor Institute, Kyoto University (KURRI-LINAC) had been originally established in 1965 by the High Voltage Engineering Co., USA and started as a $23 \mathrm{MeV}$ machine. In 1971, the machine power had been increased to $46 \mathrm{MeV}$. The KURRI-LINAC has two different operation pulse modes. One is a long mode with a maximum repetition rate of $120 \mathrm{~Hz}$, a pulse width of $0.1-4.0 \mu \mathrm{s}$ and a peak current of about $0.5 \mathrm{~A}$ for the measurement at low energies below $10 \mathrm{eV}$. Another is a short mode with a maximum repetition rate of $300 \mathrm{~Hz}$, a pulse width of $2-100 \mathrm{~ns}$ and a peak current of about $5 \mathrm{~mA}$ for the measurement at high energies above $1 \mathrm{eV}$. It is worth noting that the peak current of short mode is ten times as large as that of long mode.

By using a target according to a research purpose, we can use various type of particle beam source, i.e. neutron, electron, and photon. Thus, the research regions in the KURRI-LINAC has covered a wide field of nuclear data acquisition with the neutron Time-Of-Flight (TOF) method and a lead slowing-down spectrometer, isotope production by the $(\gamma, \mathrm{n})(\gamma, \mathrm{p})$ reaction, low-temperature electron irradiation, a photon activation analysis, and a spectroscopy with coherent $\mathrm{THz}$ radiation.

In measurements of nuclear data, a water-cooled tantalum (Ta) target as a photo-neutron target and a light water moderator has been used. There are two kinds of the moderators. One is a water tank type and another is an octagonal shape moderator called "pac-man type" as shown in Fig. 1. Researchers have usually adopted the pac-man type moderator to measure nuclear data in the past. In order to measure accurate nuclear data, it is very important to evaluate the energy resolution of a moderator. For example, the energy resolution for single

a e-mail: t-sano@rri.kyoto-u.ac.jp bunch operation at ANNRI in J-PARC had been calculated about $0.5 \%$ between energy range of $0.1 \mathrm{eV}$ from $10 \mathrm{keV}$ [1]. Detail evaluation of energy resolutions for the moderator has not carried out in KURRI-LINAC, although the energy resolution has been evaluated by using the simplified evaluation formula [2].

Thus, the energy resolutions of the neutron flux from the pac-man type moderator at the short pulse mode with $100 \mathrm{~ns}$ width were evaluated from a result of Monte Carlo simulation in this study. The researchers usually employed the electron pulse width for the nuclear data measurement in the KURRI-LINAC. Thus, the electron beam pulse width was set to $100 \mathrm{~ns}$ in this study.

\section{Monte Carlo simulation}

A calculation of the neutron flux from the pac-man type moderator was performed by the Monte Carlo code PHITS [3] with JENDL-4.0 [4] to obtain the time structure of the pulsed neuron beam. The calculational geometry is shown in Fig. 2, Fig. 3 and Fig. 4. Figure 2 shows a target assembly. The target assembly is composed of $12 \mathrm{Ta}$ plates with a total thickness of $29 \mathrm{~mm}$ in a cylindrical titanium case. Every gaps between Ta plates have $0.15 \mathrm{~mm}$ thickness. The gaps are filled with the coolant water (light water). The case has $50 \mathrm{~mm}$ diameter and $60 \mathrm{~mm}$ long. Figure 3 shows the pac-man type moderator. The light water moderator has an octagonal shape with $300 \mathrm{~mm}$ width, height and $100 \mathrm{~mm}$ thickness at the A in Fig. 3. In the center of moderator, there was a "dip" of $153 \mathrm{~mm}$ diameter and $50 \mathrm{~mm}$ thickness. There is a trade-off between increasing of neutron flux and energy resolutions. Thus, an effective thickness in the center of the pacman type moderator was set to $5 \mathrm{~cm}$ for narrow energy resolutions and a thickness at the outside of "dip" was $10 \mathrm{~cm}$ to increase the neutron flux. In addition, a lead shadow bar, $50 \mathrm{~mm}$ in diameter and $100 \mathrm{~mm}$ long, was 


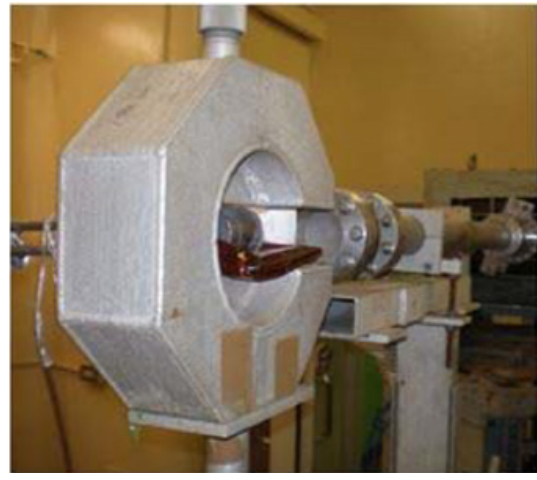

Figure 1. The pac-man type moderator and the Ta target in KURRI-LINAC.

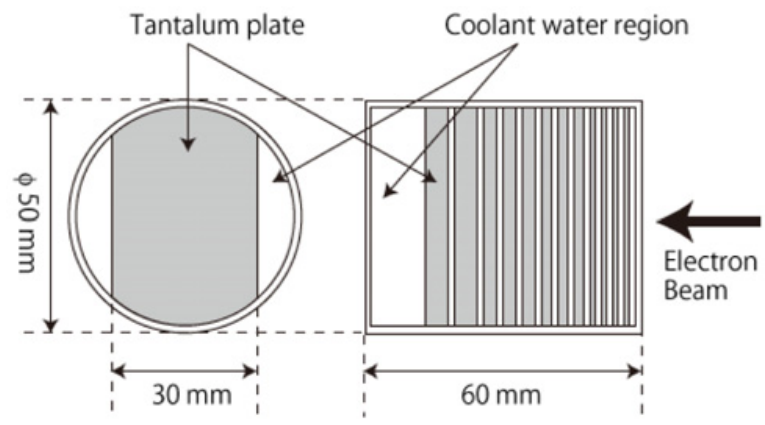

Figure 2. The tantalum target assembly.

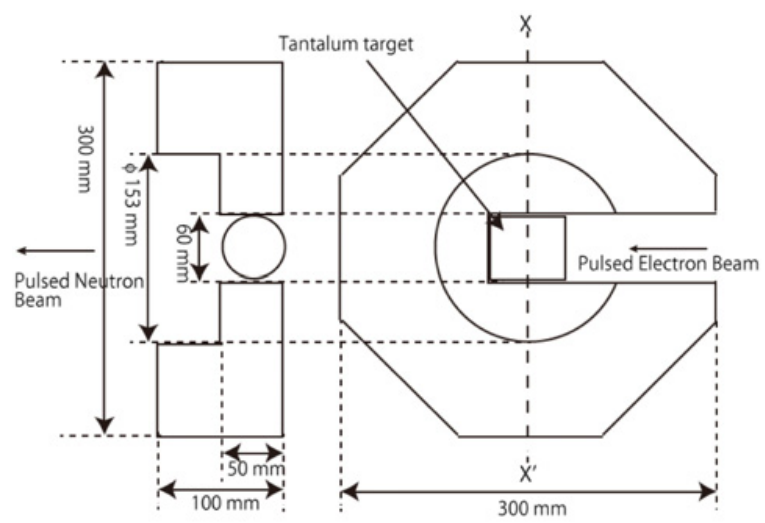

Figure 3. The pac-man type moderator. The left figure is a $\mathrm{X}-\mathrm{X}^{\prime}$ plane in the right figure.

Table 1. Parameters of the neutron flux calculation.

\begin{tabular}{|c|c|}
\hline \multicolumn{2}{|c|}{ Electron beam } \\
\hline Energy & $36 \mathrm{MeV}$ \\
\hline Diameter & $\phi 10 \mathrm{~mm}$ \\
\hline Pulse width & $100 \mathrm{nsec}$ \\
\hline
\end{tabular}

placed on the neutron beam axis in front of Ta target as shown in Fig. 4. The parameters used for the calculation are listed in Table 1. In this study, we performed the simulation including the injection of pulsed electron beam into the Ta target. The electron pules has a rectangle distribution with $100 \mathrm{~ns}$ width. A tally surface to record the neutron information was set on $35 \mathrm{~cm}$ distance from the target surface.

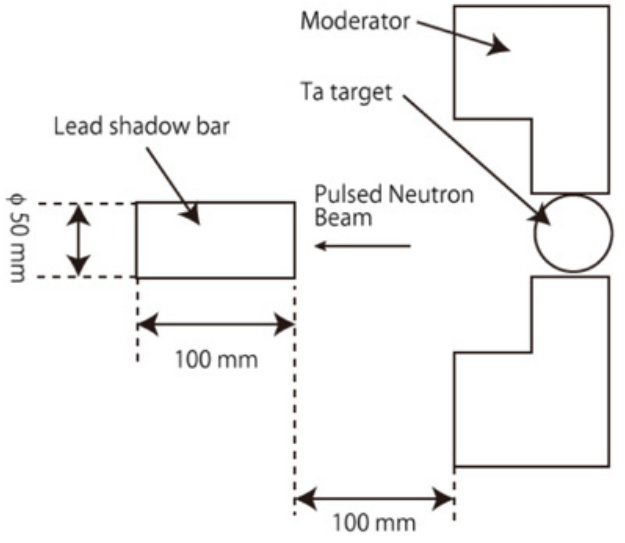

Figure 4. The Calculational geometry including the lead shadow bar.

\section{Evaluation of energy resolution}

Figure 5 shows time distributions of pulsed neutron at the tally surface obtained by the Monte Carlo simulation. The ordinate scales are linear and the peak heights are normalized to 1.0. In order to mean time structure, an expected value of time-delay is defined as following equation;

$$
\overline{\Delta t}=\frac{\sum_{i} P_{i} \Delta t_{i}}{\sum_{i} P_{i}}
$$

where, $\Delta t_{i}$ is time-delay of neutron passing through the tally surface from the injection of electron beam into the Ta target. A $P_{i}$ is probability distribution of $\Delta t_{i}$. The $P_{i}$ were obtained from the histograms shown in Fig. 5, respectively.

Energy resolution $(\Delta E / E)$ is related to the time resolution $(\Delta t / t)$ by Eq. (2),

$$
\frac{\Delta E}{E}=2 \frac{\overline{\Delta t}}{t}
$$

where, $t$ is flight time. The relation of the flight time and the neutron energy, $E(\mathrm{eV})$, is expressed as follows;

$$
t=\frac{72.298 L}{\sqrt{E}} \approx \frac{72.3 L}{\sqrt{E}},
$$

where, $L$ is a flight path (m). In nuclear data measurement at KURRI-LINAC, we usually set a sample on a flight path of $10.0 \mathrm{~m}$ or $12.7 \mathrm{~m}$ from the Ta target. In this study, the energy resolution was calculated for the $10.0 \mathrm{~m}$ TOF distance. Table 2 shows the obtained energy resolutions. The energy resolutions are about $0.7 \%$ and $0.9 \%$ between $0.1 \mathrm{eV}$ to $1 \mathrm{keV}$. On the other hand, the energy resolutions decrease above $10 \mathrm{keV}$, because the resolution has large contribution of injected electron beam with $100 \mathrm{~ns}$ pulse width.

Figure 6 shows comparison of the resolution between the results of this study and using a simplified evaluation formula. The definition of the formula is Eq. (4) [2].

$$
\frac{\Delta E}{E}=\frac{2 \sqrt{E}}{72.3 L} \sqrt{(\Delta t)^{2}+\frac{72.3^{2}}{E}(\Delta L)^{2}}=\text { const }
$$

$\Delta L$ : uncertainty of neutron flight path

$$
\Delta t=2 \sqrt{3} \tau_{m} \approx 1.85 / \sqrt{E}
$$




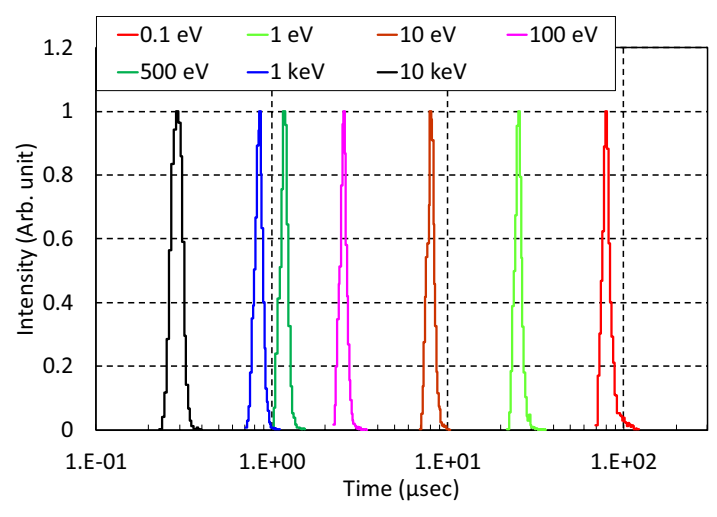

Figure 5. The time distributions of pulsed neutron at the tally surface.

Table 2. The energy resolutions with the pac-man type moderator.

\begin{tabular}{|l|c|l|c|}
\hline Energy & $\begin{array}{c}\text { Energy } \\
\text { resolution (\%) }\end{array}$ & Energy & $\begin{array}{c}\text { Energy } \\
\text { resolution (\%) }\end{array}$ \\
\hline $0.1 \mathrm{eV}$ & 0.74 & $500 \mathrm{eV}$ & 0.80 \\
\hline $1.0 \mathrm{eV}$ & 0.72 & $1 \mathrm{keV}$ & 0.93 \\
\hline $10 \mathrm{eV}$ & 0.72 & $10 \mathrm{keV}$ & 1.30 \\
\hline $100 \mathrm{eV}$ & 0.73 & \multicolumn{2}{|l}{} \\
\hline
\end{tabular}

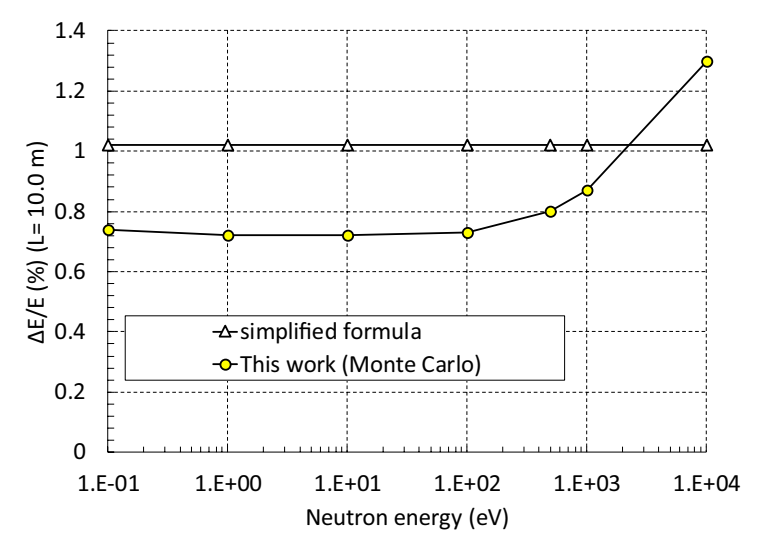

Figure 6. Comparison of energy resolution between the Monte Carlo simulation and the simplified evaluation formula.

$$
\tau_{m}=1 / \Sigma_{s} v
$$

$\Sigma s$ is a macroscopic scattering cross section of hydrogen and $v$ is a neutron velocity. A $\tau_{m}$ is a mean collision time of neutron in a moderator. In addition, we assumed the $\Delta L$ was $0.05 \mathrm{~cm}$. The flight path, $\mathrm{L}$, was $10 \mathrm{~m}$. As the results, the energy resolution was about $1.0 \%$ between $0.1 \mathrm{eV}$ and $10 \mathrm{keV}$. The energy resolution obtained from Monte Carlo simulation was agreed with the resolution using the equation (4) as shown in Fig. 6.

The obtained resolution was compared with that of the J-PARC ANNRI and the LANCSCE. Table 3 shows comparison of energy resolutions. In the ANNRI, the energy resolution at $21.5 \mathrm{~m}$ from the moderator is about
Table 3. Comparison of energy resolutions.

\begin{tabular}{|l|l|l|l|l|}
\hline \multirow{2}{*}{ Facility } & \multirow{2}{*}{$\begin{array}{l}\text { Flight } \\
\text { path }(\mathrm{m})\end{array}$} & \multicolumn{3}{|c|}{ Energy resolution (\%) } \\
\cline { 3 - 5 } & & $0.1 \mathrm{eV}-10 \mathrm{eV}$ & $100 \mathrm{eV}$ & $1 \mathrm{keV}$ \\
\hline KURRI- & 10.0 & 0.7 & 0.7 & 0.9 \\
LINAC & & & & \\
\hline LANSCE & 8.08 & 0.5 & 0.7 & 1.0 \\
\hline J-PARC AN- & 21.5 & 0.5 & $0.5^{*}$ & $0.7 *$ \\
NRI & & & $1.0^{* *}$ & $2.0^{* *}$ \\
\hline
\end{tabular}

$0.5 \%$ between energy ranges of $0.1 \mathrm{eV}$ from $10 \mathrm{eV}$ for the single- and double-bunch operation. For the double-bunch, the resolutions decrease above $10 \mathrm{eV}$ [1]. The energy resolution at the LANSCE is about $0.5 \%$ between energy ranges of $0.1 \mathrm{eV}$ from $10 \mathrm{eV}$. The resolutions decrease above $10 \mathrm{eV}$. The flight path length was $8.08 \mathrm{~m}$ [5]. The energy dependency of the energy resolution with the pacman type moderator in KURRI-LINAC is similar to the J-PARC ANNRI for the single-bunch mode.

\section{Conclusion}

In measurements of nuclear data, it is very important to evaluate the energy resolution of a moderator. Thus, the energy resolution with the pac-man type moderator at KURRI-LIAC was evaluated by using Monte Carlo simulation.

As the result, we obtained the energy resolutions $(\Delta \mathrm{E} / \mathrm{E})$ are about $0.7 \%$ to $1.3 \%$ at the energy range from $0.1 \mathrm{eV}$ to $10 \mathrm{keV}$. On the other hand, the energy resolution evaluated by the simplified formula was about $1.0 \%$ between $0.1 \mathrm{eV}$ and $10 \mathrm{keV}$. The energy resolution obtained by this study was agreed with the energy resolution evaluated by the simplified formula.

The energy dependency of the energy resolution with the pac-man type moderator in KURRI-LINAC was similar to the J-PARC ANNRI for the single-bunch mode.

In the future works, the method of energy resolution evaluation by using Monte Carlo simulation will be applied to the other moderators at KURRI-LINAC.

Present study includes the result of "Research and Development for accuracy improvement of neutron nuclear data on minor actinides" entrusted to the Japan Atomic Energy Agency by the Ministry of Education, Culture, Sports, Science and Technology of Japan (MEXT).

\section{References}

[1] K. Kino, et al., Nucl. Instrum. Methods Phys. Res. A 736, 66 (2014)

[2] O. Shcherbakov, et al., Nucl. Instrum. Methods Phys. Res. A 517, 269 (2004)

[3] T. Sato, et al., J. Nucl. Sci. Tech. 50, 913 (2013)

[4] K. Shibata, et al., J. Nucl. Sci. Tech. 48, 1 (2011)

[5] P. E. Koehler, Nucl. Instrum. Methods Phys. Res. A 350, 511 (1994) 